\title{
Burckhardtsche Bestimmung der Raumgruppen I
}

Ralph Strebel

\section{Einleitung}

Die Geometrische Kristallographie hat zum Ziel, die räumliche Verteilung der Atome, Ionen oder Moleküle eines Kristalls nach ihren Symmetrien zu klassifizieren. Um die Klassifikation eindeutig zu machen, muss man festlegen, welche räumlichen Verteilungen und welche Isometrien des Raumes zugelassen sind, und wann die Symmetriegruppen von zwei Kristallstrukturen als gleichwertig betrachtet werden. Der Mathematiker A.M. Schoenflies (1853-1928) traf in seinem 1891 erschienenen Buch Krystallsysteme und Krystallstructur die Festsetzungen, die heute noch in der Kristallographie üblich sind: als Anordnung wird jedes regelmässige Punktsystem $\mathscr{P}$ zugelassen, also jede endliche Vereinigung von Punktgittern; jede Isometrie des Raumes, die $\mathscr{P}$ auf sich abbildet, gilt als Symmetrie von $\mathscr{P}$; zwei Symmetriegruppen $G, G^{\prime}$ sind gleichberechtigt, wenn sie eigentlich affin äquivalent sind, das heisst, wenn es eine orientierungserhaltende affine Transformation $\alpha$ des Raumes gibt, derart, dass $G^{\prime}$ gleich $\alpha \cdot G \cdot \alpha^{-1}$ ist.

Schoenflies bezeichnete die Symmetriegruppen der regelmässigen Punktsysteme als Raumgruppen ([13], Kap. 6, §1) und fand 230 eigentlich affine Klassen (oder Typen) von Raumgruppen. Der Geometer und Kristallograph E.S. Fedorov (1853-1919) stiess um 1890 auf die gleichen Klassen von Symmetriegruppen, als er die regelmässigen Einteilungen des Raumes bestimmte (s. [9]).

Beide Klassifikationen werden durch geometrische Analyse der Anordnung der Symmetrie-Elemente - der Dreh- oder Schraubenachsen, der Spiegelungs-, Gleitspiegelungsoder Drehspiegelungsebenen - einer Raumgruppe gewonnen. In den Jahren 1930 bis 1936 erarbeitete dann J.J. Burckhardt ein algebraisches Bestimmungsverfahren ([4], [5] und [6]), mit dem er in seinem Lehrbuch Die Bewegungsgruppen der Kristallographie die Raumgruppen des 3-dimensionalen Raumes erneut konstruierte. Sein Verfahren erlaubt es, auch die Raumgruppen in euklidischen Räumen $E^{n}$ höherer Dimension zu bestimmen. Für $n=4$ ist diese umfangreiche Aufgabe 1973 mit Hilfe von Grossrechnern abgeschlossen worden; danach gibt es 4895 eigentlich affine Typen (s. [3]).

In diesem Artikel erläutere ich zunächst die Begriffe des Gitters und der Raumgruppe durch Beispiele und ausgewählte Ergebnisse (Abschnitte 2 und 3). Danach skizziere ich zwei Verfahren zur Konstruktion von Raumgruppen: die geometrische Methode von Schoenflies [13] und den algebraischen Zugang von Burckhardt. Da meine Skizze die 
Burckhardtsche Methode aber nicht angemessen darstellt, trage ich einige ihrer Einzelheiten in der Fortsetzung [14] dieses Aufsatzes nach.

\section{Gitter}

Gitter sind Untergruppen von euklidischen Vektorräumen mit speziellen Eigenschaften. Sie sind für die Raumgruppen aus zwei Gründen wichtig: ihre Bahnen, genannt Punktgitter, liefern den einfachsten Typ eines regelmässigen Punktsystems, und die Translationsvektoren der Translationen einer Raumgruppe bilden ein Gitter.

\subsection{Grundbegriffe}

Im folgenden bezeichnet $E=(V,\langle-,-\rangle)$ einen euklidischen Vektorraum.

Definition 2.1 Ein Gitter $\Gamma$ von $E$ ist eine diskrete Untergruppe von $E$, die ein Erzeugendensystem des Vektorraumes $V$ enthält.

Die gegebene Definition eines Gitters lässt sich etwas konkreter fassen:

Satz 2.2 Jedes Gitter $\Gamma$ in $E^{n}=\left(V^{n},\langle-,-\rangle\right)$ enthält eine Basis $\mathscr{B}=\left(b_{1}, \ldots, b_{n}\right)$ des Vektorraumes $V^{n}$, bezüglich welcher die Punkte von $\Gamma$ gerade die ganzzahligen Linearkombinationen $\sum \lambda_{i} b_{i}$ sind. Umgekehrt bilden die ganzzahligen Linearkombinationen jeder Basis von $V^{n}$ ein Gitter von E.

Beweis. Die erste Behauptung kann so begründet werden. Nach Definition enthält $\Gamma$ eine Vektorraumbasis, etwa $\mathscr{B}=\left(u_{1}, u_{2}, \ldots, u_{n}\right)$. Diese Basis gibt Anlass zu einer Fahne von Unterräumen

$$
U_{1}=\mathbb{R} u_{1}, \quad U_{2}=\mathbb{R} u_{1}+\mathbb{R} u_{2}, \quad \ldots, \quad U_{n}=V^{n} .
$$

Weil $\Gamma$ diskret ist, enthält jede Kugel $K_{r}=\{v \in V \mid\|v\| \leq r\}$ nur endlich viele Gitterpunkte. Insbesondere gibt es daher auf der Geraden $U_{1}$ einen Gitterpunkt minimaler, positiver Norm $w_{1}$; er erzeugt das Teilgitter $\Gamma \cap U_{1}$. In der Ebene $U_{2}$ betrachten wir sodann alle Geraden, die zu $U_{1}$ parallel sind und einen Gitterpunkt $x \in \Gamma \cap U_{2}$ enthalten. Jede dieser Geraden enthält einen Gitterpunkt $y \in \Gamma \cap U_{2}$, dessen Orthogonalprojektion auf $U_{1}$ höchstens die Norm $\frac{1}{2}\left\|w_{1}\right\|$ hat. Aus der Endlichkeit der Durchschnitte $\Gamma \cap K_{r}$ folgt daher, dass es in $U_{2}$ eine Gerade $w_{2}+U_{1}$ gibt, die von $U_{1}$ kleinsten, positiven Abstand aufweist. Es erzeugt dann $\left(w_{1}, w_{2}\right)$ das Teilgitter $\Gamma \cap U_{2}$. Das gegebene Argument lässt sich analog auf die Gitterpunkte in $U_{3} \backslash U_{2}, U_{4} \backslash U_{3}, \ldots$ anwenden und liefert eine Vektorraumbasis $\left(w_{1}, w_{2}, \ldots, w_{n}\right)$ von $V$, welche die Bedingung $\Gamma=\mathbb{Z} \cdot w_{1}+\mathbb{Z} \cdot w_{2}+\cdots+\mathbb{Z} \cdot w_{n}$ erfüllt. Die zweite Behauptung ergibt sich aus dem Umstand, dass jede geordnete Basis $\mathscr{B}=$ $\left(b_{1}, b_{2}, \ldots, b_{n}\right)$ von $V^{n}$ einen linearen Isomorphismus $L_{\mathscr{乃}}: \mathbb{R}^{n} \stackrel{\sim}{\longrightarrow} V$ induziert, der $\mathbb{Z}^{n}$ auf das Gitter $\Gamma$ der ganzzahligen Linearkombinationen von $\mathscr{B}$ abbildet. Da $L_{\mathscr{B}}$ und ihre Umkehrabbildung stetig sind, ist das Bild der diskreten Gruppe $\mathbb{Z}^{n} \subset \mathbb{R}^{n}$ unter $L_{\mathscr{B}}$, also $\Gamma$, eine diskrete Untergruppe von $V$.

Ebenso wie regelmässige Punktsysteme können auch Gitter Symmetrien haben. Eine für das folgende zweckmässige Definition der Symmetriegruppe eines Gitters ist diese:

Definition 2.3 Die Symmetriegruppe $\mathrm{S}(\Gamma)$ eines Gitters $\Gamma$ von $E$ besteht aus den orthogonalen Abbildungen $\varphi: E \stackrel{\sim}{\longrightarrow} E$, die $\Gamma$ auf sich abbilden. 


\subsection{Beispiele}

Seien $V=\mathbb{R}^{n}$ und $\langle-,-\rangle$ das Skalarprodukt, das dem Paar $(x, y)$ das Matrizenprodukt $x^{t} \cdot y$ zuordnet. Seien $e_{1}, e_{2}, \ldots, e_{n}$ die Standardbasisvektoren von $\mathbb{R}^{n}$. Die Gruppe $\mathbb{Z}^{n} \subset$ $\mathbb{R}^{n}$ ist diskret und enthält eine Basis von $\mathbb{R}^{n}$, sie ist also ein Gitter von $E^{n}=\left(\mathbb{R}^{n},\langle-,-\rangle\right)$. Ebenso ist jede Untergruppe von $\mathbb{Z}^{n}$, die ein Erzeugendensystem des Vektorraumes $\mathbb{R}^{n}$ enthält, ein Gitter von $\mathbb{R}^{n}$. Die folgenden Teilmengen $S_{n}, S_{n}^{\prime}$ und $S_{n}^{\prime \prime}$ erzeugen daher Gitter $\Gamma_{n}, \Gamma_{n}^{\prime}$ und $\Gamma_{n}^{\prime \prime}$ von $E^{n}$ :

$$
\begin{array}{ll}
S_{n}=\left\{ \pm 2 e_{\ell} \mid 1 \leq \ell \leq n\right\} & \text { für } n \geq 1 \\
S_{n}^{\prime}=\left\{ \pm e_{i} \pm e_{j} \mid 1 \leq i<j \leq n\right\} & \text { für } n \geq 2 \\
S_{n}^{\prime \prime}=S_{n} \cup\left\{e_{1}+e_{2}+\cdots+e_{n}\right\} & \text { für } n \geq 3
\end{array}
$$

Sie verallgemeinern die kubischen Gitter der Kristallographen.

Die Gitter $\Gamma_{2}$ und $\Gamma_{2}^{\prime}$ können wie in Fig. 1 gezeigt veranschaulicht werden.
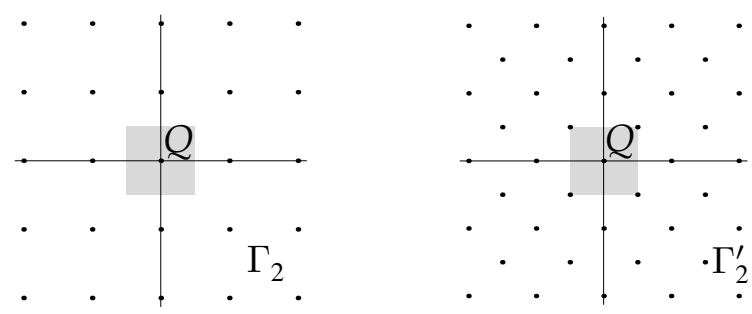

Fig. 1

Das Gitter $\Gamma_{2}$ ist quadratisch; seine Symmetriegruppe $S\left(\Gamma_{2}\right)$ fällt mit jener des Quadrates $Q=\left\{x \in \mathbb{R}^{2}|| x_{i} \mid \leq 1\right\}$ zusammen; sie besteht also aus 4 Drehungen um $(0,0)^{t}$ sowie den Spiegelungen an den Koordinatenachsen und an den Diagonalen von $Q$. Das Gitter $\Gamma_{2}^{\prime}$ ist ebenfalls quadratisch. Da $\mathrm{S}\left(\Gamma_{2}^{\prime}\right)=\mathrm{S}\left(\Gamma_{2}\right)$ ist, sind die Gitter $\Gamma_{2}$ und $\Gamma_{2}^{\prime}$ geometrisch äquivalent im Sinne der Definition 4.2. Die lineare Abbildung

$$
\sigma: \mathbb{R}^{2} \longrightarrow \mathbb{R}^{2}, \quad\left(\begin{array}{l}
x_{1} \\
x_{2}
\end{array}\right) \longmapsto \frac{1}{2}\left(\begin{array}{cc}
1 & 1 \\
1 & -1
\end{array}\right) \cdot\left(\begin{array}{l}
x_{1} \\
x_{2}
\end{array}\right)
$$

bildet das Gitter $\Gamma_{2}$ auf $\Gamma_{2}^{\prime}$ ab und induziert einen Gruppenisomorphismus

$$
\sigma_{*}: \mathrm{S}\left(\Gamma_{2}\right) \stackrel{\sim}{\longrightarrow} \mathrm{S}\left(\Gamma_{2}^{\prime}\right), \quad \varphi \longmapsto \sigma \circ \varphi \circ \sigma^{-1} .
$$

Die beiden Gitter $\Gamma_{2}$ und $\Gamma_{2}^{\prime}$ sind also sogar arithmetisch äquivalent (im Sinne der Definition 4.2). Man beachte, dass $\sigma_{*}$ die Spiegelungen an den Koordinatenachsen in jene an den Diagonalen von $Q$ überführt und daher nicht die Identität auf $S\left(\Gamma_{2}\right)=S\left(\Gamma_{2}^{\prime}\right)$ ist.

Das Gitter $\Gamma_{3}$ wird in der Kristallographie als primitiv kubisch bezeichnet. Verschiebt man mit ihm den achsenparallelen Würfel $W=\left\{x \in \mathbb{R}^{3} \mid 0 \leq x_{\ell} \leq 2\right\}$, erhält man eine Pflasterung des Raumes durch Würfel. Die Menge $S_{3}^{\prime}$ besteht aus Mittelpunkten 


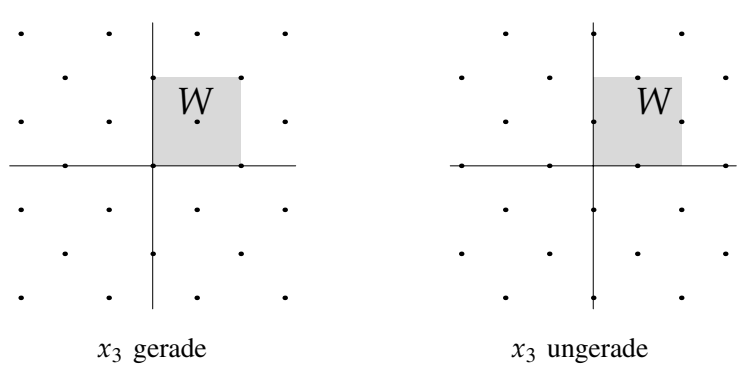

Fig. 2

der Flächen dieser Würfel, und die Rechnung $2 e_{i}=\left(e_{i}+e_{j}\right)+\left(e_{i}-e_{j}\right)$ zeigt, dass $\Gamma_{3}^{\prime}$ das primitive Gitter $\Gamma_{3}=(2 \mathbb{Z})^{3}$ umfasst. In der Kristallographie wird $\Gamma_{3}^{\prime}$ daher ein flächenzentriertes, kubisches Gitter genannt. Es wird durch Fig. 2 veranschaulicht.

In ihr sind links eine Gitterebene für einen geraden Wert von $x_{3}$ und rechts eine Gitterebene für einen ungeraden Wert von $x_{3}$ dargestellt. Beide Punktsysteme sind quadratisch, aber nicht kongruent. Dies deutet darauf hin, dass es keine Ähnlichkeitsabbildung $\sigma: \mathbb{R}^{3} \stackrel{\sim}{\longrightarrow} \mathbb{R}^{3}$ gibt, die das primitive Gitter $\Gamma_{3}$ auf das Gitter $\Gamma_{3}^{\prime}$ abbildet. Dieser Eindruck ist richtig: das Gitter $\Gamma_{3}$ hat 6 Vektoren kleinster positiver Norm, nämlich $\pm 2 e_{1}$, $\pm 2 e_{2}$ und $\pm 2 e_{3}$, während $\Gamma_{3}^{\prime}$ zwölf solche Vektoren aufweist, die Vektoren $\pm e_{i} \pm e_{j}$.

Das Gitter $\Gamma_{3}^{\prime \prime}$ besteht aus dem Teilgitter $\Gamma_{3}=(2 \mathbb{Z})^{3}$ und seiner Nebenklassse $\Gamma_{3}+$ $(1,1,1)^{t}$. Die Kristallographen nennen es ein innenzentriertes, kubisches Gitter; in der Tat bilden die Punkte der Nebenklasse $\Gamma_{3}+(1,1,1)^{t}$ die Mittelpunkte der Würfel $W+x$ mit $x \in \Gamma_{3}$. Das Gitter $\Gamma_{3}^{\prime \prime}$ besitzt 8 Punkte kleinster positiver Norm, nämlich die Punkte $\pm e_{1} \pm e_{2} \pm e_{3}$, und ist daher weder dem primitiven noch dem flächenzentrierten Gitter ähnlich. Die Symmetriegruppen der drei Gitter $\Gamma_{3}, \Gamma_{3}^{\prime}$ und $\Gamma_{3}^{\prime \prime}$ stimmen überein: die drei Gitter sind geometrisch, nicht aber arithmetisch, äquivalent (im Sinne der Definition 4.2).

\subsection{Endlichkeit der Symmetriegruppen}

Die Symmetriegruppen der Gitter von $E$ sind Untergruppen der orthogonalen Gruppe $\mathrm{O}(E)$ von $E$ mit speziellen Eigenschaften. Es gilt nämlich

Satz 2.4 Die Symmetriegruppe jedes Gitters $\Gamma$ ist endlich.

Beweis. Die Gruppe $\mathrm{S}(\Gamma)$ besteht aus den orthogonalen Abbildungen $\varphi$ von $E$, die $\Gamma$ auf sich abbilden. Diese Abbildungen bilden jede Kugel $K_{r}=\{x \in V \mid\|x\| \leq r\}$, also auch jeden Durchschnitt $\Gamma \cap K_{r}$, auf sich ab. Da diese Durchschnitte endlich sind, erhält man für jede Zahl $r>0$ durch Einschränken eine Abbildung

$$
f_{r}: \mathrm{S}(\Gamma) \longrightarrow H_{r}=\operatorname{Perm}\left(\Gamma \cap K_{r}\right)
$$

von $\mathrm{S}(\Gamma)$ in die Permutationsgruppen der endlichen Mengen $\Gamma \cap K_{r}$. Es ist $f_{r}$ ein Homomorphismus, dessen Kern aus allen $\varphi \in \mathrm{S}(\Gamma)$ besteht, die $\Gamma \cap K_{r}$ punktweise festhalten. Wählt man nun $r$ so gross, dass $\Gamma \cap K_{r}$ ein Erzeugendensystem von $V$ umfasst, wird $f_{r}$ daher injektiv. Dies zeigt, dass $S(\Gamma)$ einer Gruppe von Permutationen einer endlichen Menge isomorph ist. 
Beispiel. Wendet man obigen Beweis auf das primitive kubische Gitter $\Gamma_{3}=(2 \mathbb{Z})^{3}$ aus 2.2 an und berücksichtigt, dass jede lineare Abbildung $(0,0,0)^{t}$ festhält, erkennt man, dass $\mathrm{S}\left(\Gamma_{3}\right)$ isomorph einer Untergruppe von Perm $\left(\left\{ \pm 2 e_{1}, \pm 2 e_{2}, \pm 2 e_{3}\right\}\right)$ ist; insbesondere teilt die Ordnung von $\mathrm{S}\left(\Gamma_{3}\right)$ die Zahl $6 !=720=2^{4} \cdot 3^{2} \cdot 5$.

Bemerkungen. 1) Nach Satz 2.2 gibt es für jedes Gitter $\Gamma$ von $E^{n}$ eine geordnete Vektorraumbasis $\mathscr{B}$, die eine $\mathbb{Z}$-Basis von $\Gamma$ ist. Stellt man die Symmetriegruppe $S(\Gamma)$ in dieser Basis dar, erhält man eine endliche Untergruppe der Matrizengruppe $\operatorname{GL}(n, \mathbb{Z})$. Umgekehrt lässt sich jede endliche Untergruppe $H$ von $\operatorname{GL}(n, \mathbb{Z})$ als Symmetriegruppe eines Gitters $\Gamma \subset E^{n}$ realisieren. Zunächst ist nämlich $\mathbb{Z}^{n}$ eine diskrete Untergruppe von $\mathbb{R}^{n}$ und $H$ besteht aus Automorphismen von $\mathbb{Z}^{n}$. Mittelt man ein Skalarprodukt auf $\mathbb{R}^{n}$ mit Hilfe der Gruppe $H$, gewinnt man ein $H$-invariantes Skalarprodukt $\langle-,-\rangle_{H}$. Es sind dann $\Gamma=\mathbb{Z}^{n}$ ein Gitter von $E^{n}=\left(\mathbb{R}^{n},\langle-,-\rangle_{H}\right)$ und $H$ eine Untergruppe von $\mathrm{S}(\Gamma)$.

2) Die Ordnungen der endlichen Untergruppen $H$ von $\mathrm{GL}(n, \mathbb{Z})$ sind uniform beschränkt. Nach Minkowski ist nämlich die Einschränkung der kanonischen Projektion $\pi_{p}: \mathrm{GL}(n, \mathbb{Z}) \rightarrow \mathrm{GL}(n, \mathbb{Z} / p \mathbb{Z})$ auf $H$ injektiv, wenn $p$ eine ungerade Primzahl ist. Insbesondere besitzt $\mathrm{GL}(n, \mathbb{Z})$ nur endlich viele Isomorphietypen von endlichen Untergruppen. Es ist dies ein erstes Endlichkeitsresultat über $\mathrm{GL}(n, \mathbb{Z})$; es kann zu Theorem 8.2 (s. [14]), einem entscheidenden Hilfsmittel des Bieberbachschen Beweises von Theorem 3.5 , verschärft werden.

\section{Raumgruppen}

Die Raumgruppen treten in der Kristallographie als Gruppen der Deckbewegungen von Kristallstrukturen auf, d.h. von idealisierten Systemen der Positionen der Atome, Ionen oder Moleküle, aus denen Kristalle aufgebaut sind. In diesen Anwendungen sind die Raumgruppen also Gruppen von Deckbewegungen. Für viele Fragen ist es aber nützlich, über eine direkte Definition der Raumgruppen zu verfügen, etwa über Definition 3.1.

\subsection{Grundbegriffe}

Wie zuvor bezeichne $E=(V,\langle-,-\rangle)$ einen euklidischen Vektorraum. Eine Abbildung $\psi: E \rightarrow E$ wird Isometrie genannt, falls sie alle Distanzen erhält. Jede Isometrie ist bijektiv und die Zusammensetzung einer orthogonalen Abbildung $\varphi: E \rightarrow E$ und einer Translation $\tau_{v}$ mit Translationsvektor $v=\psi(0)$. Die Gruppe Iso $(E)$ aller Isometrien von $E$ ist, wie man leicht bestätigt, isomorph zur Produktmenge $V \times \mathrm{O}(E)$, versehen mit dem Produkt

$$
(v, \varphi) \cdot\left(v^{\prime}, \varphi^{\prime}\right)=\left(v+\varphi\left(v^{\prime}\right), \varphi \circ \varphi^{\prime}\right)
$$

Diese Formel zeigt, dass die Projektion $(v, \varphi) \mapsto \varphi$ einen Gruppenhomomorphismus $\pi$ von Iso $(E)$ auf $\mathrm{O}(E)$ liefert; sein Kern besteht aus allen Translationen von $E$.

Sei nun $G$ eine Untergruppe von Iso $(E)$. Der Kern der Einschränkung von $\pi$ auf $G$ ist der Translationsnormalteiler von $G$; ich bezeichne ihn mit $T(G)$ und die zugeordnete Untergruppe der Translationsvektoren mit $\Gamma(G)$. Die angekündigte Definition einer Raumgruppe kann nun so ausgesprochen werden:

Definition 3.1 Eine Untergruppe $G$ von Iso $(E)$ wird Raumgruppe genannt, falls $\Gamma(G)$ ein Gitter ist. 
Ist $G$ eine Raumgruppe, so bezeichnet man ihr Bild unter $\pi$ als Punktgruppe $G_{0}$ von $G$. Diese Gruppe ist isomorph zu $G / T(G)$ und endlich, denn es gilt

Hilfssatz 3.2 Die Punktgruppe $G_{0}$ einer Raumgruppe $G$ ist eine Untergruppe der Symmetriegruppe des Gitters $\Gamma(G)$.

Beweis. Es ist $T(G)$ ein Normalteiler von $G$. Die Rechnung

$$
(v, \varphi) \cdot(w, \mathbb{1}) \cdot(v, \varphi)^{-1}=(v+\varphi(w), \varphi) \cdot\left(-\varphi^{-1}(v), \varphi^{-1}\right)=(\varphi(w), \mathbb{1})
$$

impliziert deshalb, dass jede orthogonale Abbildung $\varphi \in G_{0}$ das Gitter $\Gamma(G)$ auf sich abbildet und daher in $\mathrm{S}(\Gamma(G))$ liegt.

Eine Kristallstruktur kann durch eine Funktion der Form $h: E^{3} \rightarrow \mathscr{F}$ beschrieben werden; dabei sagt der Wert $h(x)$, ob sich in $x \in E$ ein Atom oder Ion befindet, und falls ja, um welche Sorte es sich handelt. Die Invarianzgruppe $\operatorname{Inv}(h)$ einer Funktion $h: E \rightarrow \mathscr{F}$ ist ganz allgemein so erklärt:

$$
\operatorname{Inv}(h)=\{\psi \in \operatorname{Iso}(E) \mid h \circ \psi=h\} .
$$

Die Invarianzgruppe einer Funktion $h$, die ein Muster beschreibt, erlaubt es, das Muster nach seiner Regelmässigkeit zu klassifizieren. Dazu muss man aber noch festlegen, wann zwei Muster als im wesentlichen gleich betrachtet werden sollen. Zunächst wird man ein Muster und sein Bild unter einer Translation als gleichberechtigt ansehen; häufig wird man sogar das Bild des Musters unter einer Ähnlichkeitsabbildung $\sigma: E \rightarrow E$ nicht vom Original unterscheiden wollen. Beiden Festsetzungen entsprechen Konjugationsklassen von Invarianzgruppen. Wie man leicht nachrechnet, gilt nämlich die Beziehung

$$
\operatorname{Inv}\left(h \circ \sigma^{-1}\right)=\sigma \cdot \operatorname{Inv}(h) \cdot \sigma^{-1} .
$$

Obige Überlegungen veranlassen einen, auch die Raumgruppen zu Konjugationsklassen bezüglich der Translationen oder der Ähnlichkeitsabbildungen zusammenzufassen. Da die Menge der Ähnlichkeitsklassen von Raumgruppen aber immer noch unendlich ist, falls $\operatorname{dim} E>1$ beträgt, betrachtet man noch gröbere Äquivalenzrelationen, insbesondere die folgenden zwei:

Definition 3.3 Zwei Raumgruppen $G$ und $G^{\prime}$ in Iso( $(E)$ werden affin äquivalent genannt, falls es eine affine Abbildung $\alpha=\tau \circ L: E \rightarrow E$ gibt, welche die Beziehung

$$
G^{\prime}=\alpha \cdot G \cdot \alpha^{-1}=\left\{\alpha \circ \psi \circ \alpha^{-1} \mid \psi \in G\right\}
$$

erfüllt. Falls man in dieser Beziehung $\alpha$ orientierungserhaltend wählen kann, werden $G$ und $G^{\prime}$ als eigentlich affin äquivalent oder windungsäquivalent bezeichnet.

Bemerkungen. 1) Eine affine Abbildung $\alpha: E \rightarrow E$ ist die Zusammensetzung einer linearen Abbildung $L$ mit einer Translation $\tau_{v}: x \mapsto x+v$; eine Raumgruppe $G$ besteht dagegen aus Isometrien. Ist nun $\alpha: E \rightarrow E$ eine bijektive affine Abbildung, so wird $\alpha \cdot G \cdot \alpha^{-1}$ im allgemeinen keine Raumgruppe sein. Ist sie es, so zeigt die Rechnung

$$
(v, L) \cdot(w, \varphi) \cdot(v, L)^{-1}=\left(L(w)+v-L \circ \varphi \circ L^{-1}(v), L \circ \varphi \circ L^{-1}\right),
$$

dass $L \circ \varphi \circ L^{-1}$ für jede orthogonale Abbildung $\varphi \in G_{0}$ orthogonal sein muss. Dies bedingt, dass $L$ der Gruppe $G_{0}$ angepasst ist; genauer gilt der 
Satz 3.4 Sind $G_{0}$ eine Untergruppe von $\mathrm{O}(E)$ und $L: E \stackrel{\sim}{\longrightarrow} E$ eine bijektive lineare Abbildung mit $L \cdot G_{0} \cdot L^{-1} \subset \mathrm{O}(E)$, so gibt es eine lineare, symmetrische und positive Abbildung $P$, die mit allen $\varphi \in G_{0}$ vertauschbar ist, und eine orthogonale Abbildung $\psi$, welche die Bedingungen $L=\psi \circ P$ und $L \cdot G_{0} \cdot L^{-1}=\psi \cdot G_{0} \cdot \psi^{-1}$ erfüllen.

2) Sind $G$ und $G^{\prime}$ affin äquivalent vermöge $\alpha=\tau \circ L$, so zeigt die Rechnung (3.5), dass die Paare $\left(\Gamma(G), G_{0}\right)$ und $\left(\Gamma\left(G^{\prime}\right), G_{0}^{\prime}\right)$ die Bedingung

$$
L(\Gamma)=\Gamma^{\prime} \text { und } L \cdot G_{0} \cdot L^{-1}=G_{0}^{\prime}
$$

erfüllen. Diese Bedingung definiert eine Relation, welche die affine Äquivalenz abschwächt; sie kommt in Abschnitt 4 ausführlicher zur Sprache.

\subsection{Beispiele}

1) Invarianzgruppe eines Gitters. Seien $\Gamma \subset E$ ein Gitter und $h: E \rightarrow\{0,1\}$ die charakteristische Funktion $\chi_{\Gamma}$ des Gitters (es ist also $\chi_{\Gamma}(x)=1 \Leftrightarrow x \in \Gamma$ ). Die Invarianzgruppe von $\chi_{\Gamma}$ enthält dann die Translationen $\tau_{v}$ mit $v \in \Gamma$ und die orthogonalen Abbildungen $\varphi \in \mathrm{S}(\Gamma)$, und sie wird von ihnen erzeugt; genauer ist

$$
\operatorname{Inv}\left(\chi_{\Gamma}\right)=\left\{\tau_{v} \circ \varphi \mid \varphi \in \mathrm{S}(\Gamma), v \in \Gamma\right\} \stackrel{\sim}{\longrightarrow} \Gamma \rtimes \mathrm{S}(\Gamma)
$$

Sei $\Gamma^{\prime}$ ein zweites Gitter in $E$. Wenn die Gruppen $G=\operatorname{Inv}\left(\chi_{\Gamma}\right)$ und $G^{\prime}=\operatorname{Inv}\left(\chi_{\Gamma^{\prime}}\right)$ affin äquivalent sind, so gibt es definitionsgemäss eine bijektive Abbildung $\alpha=\tau \circ L: E \stackrel{\sim}{\longrightarrow} E$ mit $\alpha \cdot G \cdot \alpha^{-1}=G^{\prime}$. Eine kurze Rechnung zeigt, dass dies genau dann gilt, falls $\Gamma^{\prime}=L(\Gamma)$ und $\mathrm{S}\left(\Gamma^{\prime}\right)=L \cdot \mathrm{S}(\Gamma) \cdot L^{-1}$ ist, falls also die Paare $(\Gamma, \mathrm{S}(\Gamma))$ und $\left(\Gamma^{\prime}, \mathrm{S}\left(\Gamma^{\prime}\right)\right)$ die Bedingung (3.6) erfüllen.

2) Raumgruppe der Elemente vom Kupfertyp. Die Atome des Metalles Kupfer bilden ein flächenzentriertes kubisches Punktgitter $F \in \mathbb{R}^{3}$, das dem Gitter $\Gamma_{3}^{\prime}=\left\{x \in \mathbb{Z}^{3} \mid\right.$ $x_{1}+x_{2}+x_{3}$ gerade $\}$ ähnlich ist (s. Fig. 3, links). Die Invarianzgruppe von $\chi_{F}$ ist daher affin äquivalent zur Gruppe $\operatorname{Inv}\left(\chi_{\Gamma_{3}^{\prime}}\right) \stackrel{\sim}{\longrightarrow} \Gamma_{3}^{\prime} \rtimes \mathrm{S}\left(\Gamma_{3}^{\prime}\right)$.

Die Gruppe $\mathrm{S}\left(\Gamma_{3}^{\prime}\right)$ ist die Symmetriegruppe des Würfels $W^{\prime}=\left\{x \in \mathbb{R}^{3}|| x_{i} \mid \leq 1\right\}$; sie besitzt 48 Elemente und wird in der Kristallographie mit $O_{h}$ oder $\frac{4}{m} \frac{2}{m}$ bezeichnet. ${ }^{1)}$ Der erste Teil des Symbols $\frac{4}{m} \overline{3} \frac{2}{m}$, also $\frac{4}{m}$, besagt, dass das Gitter Drehungen der Ordnung 4 um die Koordinatenachsen und Spiegelungen an den Koordinatenebenen zulässt; das Symbol $\overline{3}$ deutet darauf hin, dass die Raumdiagonale $\mathbb{R} \cdot(1,1,1)^{t}$ die Achse einer Drehspiegelung der Ordnung 6 ist; schliesslich zeigt $\frac{2}{m}$, dass die Achsen durch die Mittelpunkte gegenüberliegender Kanten 2-zählige Drehachsen sind und das Gitter Spiegelungen an Ebenen, die auf diesen Drehachsen senkrecht stehen, zulässt. Der Typ der Raumgruppe $\operatorname{Inv}\left(\Gamma_{3}^{\prime}\right)$ wird mit $F \frac{4}{m} \overline{3} \frac{2}{m}$ bezeichnet; s. [11]. Die Kristallstrukturen der Metalle $\mathrm{Al}, \mathrm{Ag}, \mathrm{Au}$ und $\mathrm{Pb}$ sind jener des Kupfers ähnlich; ihre Raumgruppen sind daher vom gleichen Typ.

1) Die Kristallographen bezeichnen die geometrischen Kristallklassen auf zwei Weisen. Die erste geht auf Schoenflies zurück; bei ihr trägt die Symmetriegruppe des Würfels oder regelmässigen Oktaeders die Bezeichnung $\mathrm{O}_{h}$. Die andere Bezeichnungsweise stammt von Hermann und Mauguin; sie ist sprechender, aber gewöhnungsbedürftig. 


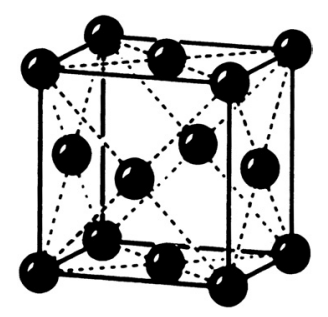

Kristallstruktur von $\mathrm{Cu}$

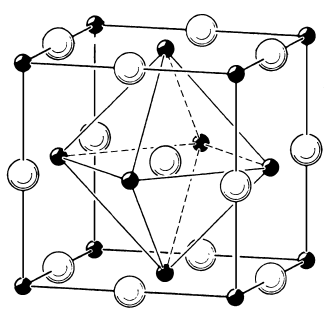

Kristallstruktur von $\mathrm{NaCl}$

Fig. 3

3) Raumgruppen der Verbindungen vom Typ $\mathrm{NaCl}$. Beim Steinsalz bilden die Natriumionen $\mathrm{Na}^{+}$ein flächenzentriertes kubisches Punktgitter $F^{+}$, die Chlorionen $\mathrm{Cl}^{-}$ein zu $\mathrm{F}^{+}$ kongruentes Punktgitter $F^{-}$. Diese Anordnung ist dem Paar $\left(\Gamma_{3}^{\prime}, \Gamma_{3}^{\prime}+(1,1,1)^{t}\right)$ ähnlich; sie ist in Fig. 3 rechts dargestellt. Die Raumgruppe ist erneut vom Typ $F \frac{4}{m} \overline{3} \frac{2}{m}$.

\subsection{Endlichkeit der Typen von Raumgruppen}

Die in Definition 3.3 festgelegten Relationen der affinen und der eigentlich affinen Äquivalenz führen im Falle des 3-dimensionalen Raumes zu endlich vielen Klassen. Dies wurde um 1890 von E.S. Fedorov und von A. Schoenflies bewiesen, und zwar durch explizite Bestimmung der Typen: bezüglich der eigentlich affinen Äquivalenz gibt es 230 Typen von Raumgruppen.

Die grosse Anzahl der Typen macht es schwierig, vorher zu sagen, wie das Ergebnis für höherdimensionale Räume lautet. D. Hilbert warf deshalb in der Ausarbeitung seines Pariser Vortrages von 1900 die folgende Frage auf (Problem 18 in [10]):

[...] es ist daher die Untersuchung der Frage wünschenswert, ob es auch im n-dimensionalen Euklidischen Raume nur eine endliche Anzahl wesentlich verschiedener Arten von Bewegungsgruppen mit Fundamentalbereich gibt.

1910 schon konnte L. Bieberbach seine Antwort ankündigen; er begründete sie in den Arbeiten [1] und [2]:

Theorem 3.5 Für jeden euklidischen Raum E enthält Iso(E) nur endlich viele Typen von Raumgruppen.

Die Beweise von Theorem 3.5 - etwa jener in [8] - liefern selbst in kleinen Dimensionen astronomisch grosse Schranken. Die Tabelle unten zeigt zum Vergleich die heute bekannten, exakten Anzahlen.

\section{Konstruktion der Raumgruppen - Skizze}

Die Aufgabe, die Raumgruppen eines gegebenen euklidischen Raumes bis auf affine Äquivalenz zu bestimmen, ist umfangreich, falls $\operatorname{dim} E>2$ ist. Es ist daher naheliegend, in einem ersten Schritt Klassifikationen nach Äquivalenzrelationen, die jene der affinen oder eigentlich affinen Äquivalenz vergröbern, vorzunehmen. 
Seien $G$ und $G^{\prime}$ Raumgruppen von $E$ mit Gittern $\Gamma=\Gamma(G), \Gamma^{\prime}=\Gamma\left(G^{\prime}\right)$ und Punktgruppen $G_{0}, G_{0}^{\prime}$. Man nennt $G$ und $G^{\prime}$ arithmetisch äquivalent, falls es eine affine Transformation $\alpha=\tau \circ L: E \rightarrow E$ gibt, welche die Bedingung (3.6) erfüllt. Diese Bedingung ist nur eine Forderung an die Paare $\left(\Gamma, G_{0}\right)$ und $\left(\Gamma^{\prime}, G_{0}^{\prime}\right)$; sie besagt, dass die Paare arithmetisch äquivalent im Sinne der Definition 4.1 sind:

Definition 4.1 Zwei Paare $(\Gamma, H \subseteq \mathrm{S}(\Gamma))$ und $\left(\Gamma^{\prime}, H^{\prime}\right)$ werden arithmetisch äquivalent genannt, falls es eine lineare Bijektion $L: E \stackrel{\sim}{\longrightarrow} E$ gibt, welche die Bedingung

$$
\Gamma^{\prime}=L(\Gamma) \quad \text { und } \quad H^{\prime}=L \cdot H \cdot L^{-1}
$$

erfüllt. Genügt $L$ der schwächeren Bedingung $H^{\prime}=L \cdot H \cdot L^{-1}$, bezeichnet man die Paare als geometrisch äquivalent.

Die Äquivalenzklassen der arithmetischen oder geometrischen Äquivalenz nennt man die arithmetischen oder die geometrischen Kristallklassen (oder Klassen). Wie die folgende Tabelle vor Augen führt, ist die Mächtigkeit der Menge der arithmetischen Klassen $\mathscr{A}$ und jene der Menge der geometrischen Klassen $\mathscr{G}$ deutlich kleiner als die Anzahl der affinen Typen von Raumgruppen. (Die Zahlen stammen für $n \leq 4$ aus [3], S. 52, und für $n \in\{5,6\}$ aus [12], Tabelle 2).

\begin{tabular}{|c|c|c|c|}
\hline $\operatorname{dim} E$ & Raumgruppentypen & $\operatorname{card}(\mathscr{A})$ & $\operatorname{card}(\mathscr{G})$ \\
\hline \hline 2 & 17 & 13 & 10 \\
3 & 219 & 73 & 32 \\
4 & 4783 & 710 & 227 \\
5 & 222018 & 6079 & 955 \\
6 & 28927922 & 85311 & 7014 \\
\hline
\end{tabular}

Definition 4.1 liefert auch Einteilungen der Gitter:

Definition 4.2 Zwei Gitter $\Gamma$ und $\Gamma^{\prime}$ werden arithmetisch äquivalent genannt, falls die Paare $(\Gamma, S(\Gamma))$ und $\left(\Gamma^{\prime}, S\left(\Gamma^{\prime}\right)\right)$ arithmetisch äquivalent sind. Analog definiert man die geometrische Äquivalenz von Gittern.

\subsection{Bestimmung der geometrischen Kristallklassen von $E^{3}$}

Die Bestimmung der geometrischen Kristallklassen läuft nach Definition 4.1 und Satz 3.4 darauf hinaus, alle endlichen Untergruppen der orthogonalen Gruppe $\mathrm{O}(E)$ zu finden, die in einer geeigneten Basis ganzzahlig dargestellt werden, und diese Untergruppen bis auf Konjugation in $\mathrm{O}(E)$ zu klassifizieren.

Im Falle des 3-dimensionalen Raumes ist diese Bestimmung bereits in der ersten Hälfte des 19. Jahrhunderts durchgeführt worden. Sie kann so erfolgen: man klassifiziert zunächst alle endlichen Untergruppen von $\mathrm{O}(3, \mathbb{R})$ bis auf Konjugation und entfernt dann aus dieser Liste jene Gruppen, die keine treue ganzzahlige Darstellung in $\operatorname{GL}(3, \mathbb{Z})$ haben. Beide Teile dieses Weges sind recht kurz; dass dem so ist, beruht auf Eigenschaften von $E^{3}$, die in höheren Dimensionen nicht mehr vorliegen. 
Endliche Untergruppen von $\mathrm{O}(3, \mathbb{R})$. Die Suche der endlichen Untergruppen $H$ von $\mathrm{SO}(3, \mathbb{R})$ wird durch den Umstand erleichtert, dass jedes Element $\varphi \in H \backslash\{\mathbb{\|}\}$ eine Drehung um eine Achse ist, welche die Einheitssphäre $\mathbb{S}^{2}$ in zwei Punkten durchstösst, den Polen von $\varphi$. Die Menge aller Pole ist eine Teilmenge $\mathscr{P} \subset \mathbb{S}^{2}$, auf der $H$ operiert. Analysiert man diese Wirkung, kommt man zu

Satz 4.3 Für jede endliche Untergruppe $H \subset \mathrm{SO}(3, \mathbb{R})$ trifft eine der folgenden Aussagen zu:

(i) Alle Drehachsen von $H \backslash\{\|\}$ stimmen überein. Dann ist $H$ zyklisch.

(ii) Es gibt genau 3 Drehachsen; sie sind paarweise orthogonal und $H$ ist isomorph der Gruppe $\mathbb{Z} / 2 \mathbb{Z} \times \mathbb{Z} / 2 \mathbb{Z}$.

(iii) Es gibt mehr als 3 Drehachsen und auf einer unter ihnen stehen alle anderen senkrecht. $H$ ist dann eine Diedergruppe der Ordnung $2 m$ mit $m>2$.

(iv) Die Pole der 3-zähligen Drehachsen bilden die Ecken eines Würfels W oder eines Dodekaeders D. Dann ist $H$ die Drehgruppe der dem Würfel $W$ einbeschriebenen Tetraeder, die Drehgruppe von $W$ oder jene von $D$.

Die Untergruppen von $\mathrm{O}(3, \mathbb{R})$ ergeben sich leicht aus jenen von $\mathrm{SO}(3, \mathbb{R})$. Es ist nämlich $\operatorname{det}(-\mathbb{1})=(-1)^{3}=-1$ und daher ist $\mathrm{O}(3, \mathbb{R})$ das direkte Produkt der Untergruppen $\mathrm{SO}(3, \mathbb{R})$ und $\overline{1}=\{\mathbb{1},-\mathbb{\|}\}$. Jede Untergruppe $U$ von $\mathrm{O}(3, \mathbb{R})$ gehört daher zu einer von 3 Sorten: (a) $U \subseteq \operatorname{SO}(3, \mathbb{R})$, (b) $-\mathbb{1} \in U$ oder (c) $U \subsetneq \operatorname{SO}(3, \mathbb{R})$ und $-\mathbb{1} \notin U$. Im zweiten Fall ist $U=(U \cap \mathrm{SO}(3, \mathbb{R})) \times \overline{1}$; im dritten Fall ist der offensichtliche Homomorphismus $U \hookrightarrow \mathrm{SO}(3, \mathbb{R}) \times \overline{1} \rightarrow \mathrm{SO}(3, \mathbb{R})$ injektiv; $U$ ist also isomorph einer Untergruppe $\hat{U} \subset \mathrm{SO}(3, \mathbb{R})$ und entsteht aus $\hat{U}$ dadurch, dass jedes Element $\varphi$ von $\hat{U} \backslash(U \cap \mathrm{SO}(3, \mathbb{R}))$ durch $-\varphi$ ersetzt wird.

Kristallographische Untergruppen von $\mathrm{O}(3, \mathbb{R})$. Das Aussondern der endlichen Untergruppen von $\mathrm{O}(3, \mathbb{R})$, die nicht kristallographisch sind, also kein Gitter invariant lassen, geschieht wie folgt. Sei $\varphi$ eine Drehung in $E^{3}$, die ein Gitter $\Gamma$ von $E^{3}$ auf sich abbildet. Berechnet man die Spur von $\varphi$ in einer orthonormierten Basis $\mathscr{B}=\left(b_{1}, b_{2}, b_{3}\right)$ mit $\varphi\left(b_{1}\right)=b_{1}$, sieht man, dass $\operatorname{sp} \varphi=1+2 \cos t$ ist; wählt man hingegen eine Basis von $E^{3}$, die eine $\mathbb{Z}$-Basis des Gitters $\Gamma$ ist, so erkennt man, dass $\operatorname{sp} \varphi$ in $\mathbb{Z}$ liegt. Folglich ist $\cos t \in\left\{1, \frac{1}{2}, 0,-\frac{1}{2},-1\right\}$ und $\varphi$ hat die Ordnung 1, 6, 4, 3 oder 2. Diese Folgerung nennt man die kristallographische Bedingung an die Rotationen von $E^{3}$.

Sei nun $H$ eine endliche Untergruppe von $\mathrm{SO}\left(E^{3}\right)$, die ein Gitter $\Gamma$ invariant lässt. Jede der Drehungen $\varphi \in H$ hat dann eine der Ordnungen 1, 2, 3, 4 oder 6. Das schliesst die meisten der in Satz 4.3 aufgezählten Gruppen aus. Übrig bleiben die zyklischen Gruppen der Ordnungen 1, 2, 3, 4 und 6, die Diedergruppen der Ordnungen 4, 6, 8 und 12, sowie die Drehgruppen eines regulären Tetraeders oder Oktaeders. Diese Gruppen vertreten 11 verschiedene Konjugationsklassen. Bildet man die direkten Produkte der genannten Gruppen mit $\overline{1}=\{\mathbb{1},-\mathbb{1}\}$, erhält man Vertreter von 11 weiteren Konjugationsklassen. Es verbleibt die Aufgabe, in den Vertretern $\hat{H}$ der ersten 11 Klassen Untergruppen $N$ vom Index 2 aufzusuchen, und die Gruppen $H=N \cup\{-\varphi \mid \varphi \in \hat{H} \backslash N\}$ zu klassifizieren. Dies führt auf 10 weitere Klassen. Insgesamt kommt man zu $11+11+10=32$ Konjugationsklassen kristallographischer Gruppen oder (geometrischen) Kristallklassen. 
Beispiel. Sei $\hat{H}$ die Drehgruppe eines Würfels; sie hat die Ordnung 24, ist isomorph der symmetrischen Gruppe $S_{4}$ und besitzt genau einen Normalteiler $N$ vom Index 2; dieser ist isomorph der alternierenden Gruppe $A_{4}$. Aus $\hat{H}$ und $N$ gewinnt man dann 5 Gruppen, nämlich

$$
N, \quad \hat{H}, \quad N \times \overline{1}, \quad H=N \cup-(\hat{H} \backslash N) \quad \text { und } \quad \hat{H} \times \overline{1} .
$$

Sie vertreten die Kristallklassen des sogenannten kubischen Kristallsystems. In der Schoenfliesschen Notation werden sie mit $T, O, T_{h}, T_{d}$ und $O_{h}$ bezeichnet, in jener von Hermann-Mauguin mit

$$
\text { 233, 432, } \frac{2}{m} \overline{3} \text { oder } m \overline{3}, \quad \overline{4} 3 m \text {, und } \frac{4}{m} \overline{3} \frac{2}{m} \text { oder } m \overline{3} m \text {. }
$$

Vier dieser Klassen werden durch Symmetriegruppen von regelmässigen Polyedern vertreten. Die Drehgruppe $\mathrm{S}^{+}(\mathcal{T})$ eines regelmässigen Tetraeders $\mathcal{T}$ (mit Zentrum $(0,0,0)^{t}$ ) ist vom Typ $T \sim 233$; die volle Symmetriegruppe $S(\mathscr{T})$ vom Typ $T_{d} \sim \overline{4} 3 m$, denn $S(\mathscr{T})$ enthält zwar Spiegelungen, nicht aber -11 . Das Symbol $\overline{4}$ in der Bezeichnung $\overline{4} 3 m$ drückt aus, dass $S(\mathscr{T})$ eine Drehspiegelung der Ordnung 4 enthält. Ist 0 ein reguläres Oktaeder, so gehört $\mathrm{S}^{+}(\mathcal{O})$ zur Klasse mit dem Namen $O$ oder 432, und $\mathrm{S}(\mathcal{O})$ zur Klasse $O_{h}$ oder $\frac{4}{m} \overline{3} \frac{2}{m}$.

Es verbleibt die Klasse $T_{h}$ oder $\frac{2}{m} \overline{3}$. Das Symbol $T_{h}$ deutet darauf hin, dass ein geeigneter Vertreter eine horizontale Spiegelungsebene enthält. Polyeder mit dieser Symmetrie sind die in Fig. 4 dargestellten Dodekaeder.
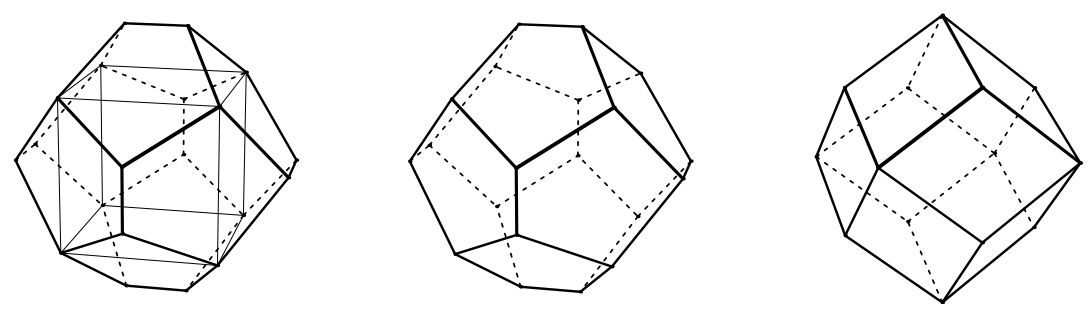

Fig. 4

Die Figur links erklärt ihre Konstruktion: man setzt auf jede Seitenfläche eines Würfels ein Walmdach und zwar so, dass die Neigungen der Flächenstücke, die längs einer Kante aneinander stossen, übereinstimmen und dass das so entstehende Dodekaeder 3-zählige Drehungen um die Diagonalen des Würfels zulässt. Das Dodekaeder besitzt dann 12 kongruente fünfeckige Flächen mit 4 gleichlangen Kanten. Hat die fünfte Kante eine andere Länge, so ist $\mathrm{S}(\mathscr{P})$ vom Typ $T_{h}$. Dies gilt auch dann noch, wenn die fünfte Kante die Länge 0 hat: $\mathscr{P}$ ist dann ein Rhombendodekaeder.

\subsection{Bestimmung der arithmetischen Kristallklassen des $E^{3}$}

Die arithmetischen Kristallklassen von $E$ können mit verschiedenen Verfahren gefunden werden. Eines geht auf das 19. Jahrhundert zurück: bei ihm gewinnt man die arithmetischen Klassen durch Verfeinerung der geometrischen Klassen (s. anschliessendes Beispiel). Bei einem zweiten Verfahren bestimmt man Vertreter der arithmetischen Klassen auf direktem Wege (s. [14], Nummer 8.3). 
Beispiel. Seien $H$ eine endliche Untergruppe von $\mathrm{SO}\left(E^{3}\right)$ vom Typ $C_{4}$ und $\rho$ eine Drehung mit Drehachse $U$, die $H$ erzeugt. Sei weiter $\Gamma$ ein Gitter von $E^{3}$, das von $H$ auf sich abgebildet wird. Es gibt einen Gitterpunkt $v \in \Gamma \backslash U$. Mit $v$ sind auch $\rho(v)$ und $w=v-\rho(v)$ Gitterpunkte; da $w \neq(0,0,0)^{t}$ ist und auf $U$ senkrecht steht, enthält die Drehebene $W=U^{\perp}$ einen Gitterpunkt positiver Länge. Es folgt, dass $\Gamma \cap W$ ein quadratisches Gitter von $W$ ist; seien $b_{1}, b_{2}$ orthogonale Vektoren gleicher Länge, die $\Gamma \cap W$ erzeugen. Die Summe $v+\rho(v)+\rho^{2}(v)+\rho^{3}(v)$ ist ungleich $(0,0,0)^{t}$ und wird von $\rho$ festgehalten. Also ist $U \cap \Gamma$ ein Gitter in $U$; insbesondere gibt es einen Vektor $b_{3} \in \Gamma \cap U$ mit kleinster positiver Norm.

Zwei Fälle treten nun auf: $\Gamma$ ist entweder gleich $\Gamma_{1}=(\Gamma \cap W) \oplus(\Gamma \cap U)$, oder es gibt einen Vektor $u \in \Gamma \backslash \Gamma_{1}$. Im zweiten Fall ist $(\Gamma \cap W)+u$ eine Teilmenge der affinen Ebene $W+u$, die unter der Drehung $\rho$ invariant ist. Dies ist nur möglich, wenn die Orthogonalprojektion von $(\Gamma \cap W)+u$ auf die Ebene $W$ mit der Menge $(\Gamma \cap W)+\frac{1}{2}\left(b_{1}+b_{2}\right)$ zusammenfällt. Es gehört dann $2 \cdot u$ zu $\Gamma_{1}$ und $\Gamma$ ist gleich $\Gamma_{1} \cup \Gamma_{1}+\frac{1}{2}\left(b_{1}+b_{2}+b_{3}\right)$ : das Gitter $\Gamma$ entsteht aus $\Gamma_{1}$ durch Zentrierung.

Dieses Ergebnis bedeutet, dass die geometrische Kristallklasse $C_{4}$ aus genau zwei arithmetischen Kristallklassen zusammengesetzt ist. Sei nämlich $L: E^{3} \rightarrow \mathbb{R}^{3}$ die lineare Abbildung, die der geordneten Basis $\left(b_{1}, b_{2}, b_{3}\right)$ von $E^{3}$ die Standardbasis $\left(e_{1}, e_{2}, e_{3}\right)$ von $\mathbb{R}^{3}$ zuordnet. Durch $L$ wird $\Gamma_{1}$ auf das Standardgitter $\mathbb{Z}^{3}$ von $\mathbb{R}^{3}$ geworfen; da die Vektoren $b_{1}, b_{2}$ und $b_{3}$ paarweise orthogonal sind und $b_{1}, b_{2}$ gleiche Länge haben, ist $\rho^{\prime}=L \cdot \rho \cdot L^{-1}$ eine Drehung um die Achse $\mathbb{R} \cdot e_{3}$. Sei $H^{\prime} \subset \operatorname{SO}(3, \mathbb{R})$ die von $\rho^{\prime}$ erzeugte Untergruppe. Im ersten Fall ist das Paar $(\Gamma, H)$ dem Paar $\left(\mathbb{Z}^{3}, H^{\prime}\right)$ arithmetisch äquivalent, im zweiten dem Paar $\left(\mathbb{Z}^{3} \cup \mathbb{Z}^{3}+\frac{1}{2}(1,1,1)^{t}, H^{\prime}\right)$. Die beiden Paare sind nicht arithmetisch äquivalent: es wird nämlich $\mathbb{Z}^{3}$ von Gitterpunkten erzeugt, die auf der Achse $U^{\prime}=\mathbb{R} \cdot e_{3}$ und der Drehebene $W^{\prime}=\left(U^{\prime}\right)^{\perp}$ liegen, nicht aber $\mathbb{Z}^{3} \cup \mathbb{Z}^{3}+\frac{1}{2}(1,1,1)^{t}$.

\subsection{Konstruktion der Raumgruppen nach Schoenflies}

In dieser und der folgenden Nummer vergleiche ich die Konstruktionen von Schoenflies und Burckhardt am Beispiel der Raumgruppen der geometrischen Klasse $C_{4}$.

Seien $G$ eine Raumgruppe von $E^{3}$, deren Punktgruppe $H=G_{0}$ von einer Drehung der Ordnung 4 erzeugt wird, $U$ die Achse dieser Drehung und $\pi_{U}: E^{3} \rightarrow E^{3}$ die Orthogonalprojektion auf diese Achse. In $G$ gibt es dann eine Isometrie der Form $\left(v_{\varphi}, \varphi\right)$; sie ist eine Drehung, wenn $\pi_{U}\left(v_{\varphi}\right)$ der Nullvektor ist, und sonst eine Schraubung. In jedem Falle liegt $4 \cdot \pi_{U}\left(v_{\varphi}\right)$ in $\Gamma=\Gamma(G)$. Durch Verschieben des Ursprungs kann man erreichen, dass $v_{\varphi}$ und $\pi_{U}\left(v_{\varphi}\right)$ zusammenfallen. Nach Nummer 4.2 gibt es eine Basis $\mathscr{B}=\left(b_{1}, b_{2}, b_{3}\right)$ von $E^{3}$, so dass $b_{3}$ das Teilgitter $\Gamma \cap U$ von $U$ und $b_{1}, b_{2}$ das Teilgitter $\Gamma \cap W$ von $W=U^{\perp}$ erzeugen. Wir können annehmen, $b_{1}$ und $b_{2}$ seien orthogonal und von der gleichen Länge. Dann ist entweder $\Gamma$ gleich dem Teilgitter $\Gamma_{1}=(\Gamma \cap W) \oplus(\Gamma \cap U)$, oder gleich $\Gamma_{1} \cup \Gamma_{1}+\frac{1}{2}\left(b_{1}+b_{2}+b_{3}\right)$.

Die Gruppe $G$ enthält mit $\left(v_{\varphi}, \varphi\right)$ auch jedes Paar der Form $\left(w+v_{\varphi}, \varphi\right)$ mit $w \in \Gamma$. Um die Achsen dieser Paare zu finden, suchen wir die Vektoren $x \in W$, welche die Gleichung $\varphi(x)+\pi_{W}(w)+v_{\varphi}=x+v_{\varphi}$ oder $\pi_{W}(w)=(\mathbb{1}-\varphi)(x)$ erfüllen. Sei $L_{\varphi}: W \rightarrow W$ die lineare Abbildung $(\mathbb{1}-\varphi)_{\mid W}$. Wir können annehmen, $L_{\varphi}$ werde bezüglich $\left(b_{1}, b_{2}\right)$ 
durch die Matrix

$$
A=\left(\begin{array}{ll}
1 & 0 \\
0 & 1
\end{array}\right)-\left(\begin{array}{cc}
0 & -1 \\
1 & 0
\end{array}\right)=\left(\begin{array}{cc}
1 & 1 \\
-1 & 1
\end{array}\right)
$$

beschrieben; $A$ ist regulär. Die Umkehrabbildung $L_{\varphi}^{-1}$ ist dann eine Drehstreckung mit Drehwinkel $\pi / 4$ und Faktor $\sqrt{2} / 2$.

Wir unterscheiden nun zwei Fälle. Ist $\Gamma=\Gamma_{1}$, also ein primitives Gitter, so ist $\pi_{W}(\Gamma)=$ $\Gamma \cap W=\mathbb{Z} b_{1} \oplus \mathbb{Z} b_{2}$, weshalb $G$ eine Schar von Achsen aufweist, die $W$ in dem quadratischen Gitter $L_{\varphi}^{-1}(\Gamma \cap W)$ durchstossen. Je nach dem Wert von $v_{\varphi}$ gehören diese Achsen zu Drehungen oder Schraubungen. Ihre geometrische Verteilung ist in Fig. 5 für $v_{\varphi}=\frac{k}{4} b_{3}$ und $k \in\{0,1,2,3\}$ dargestellt.

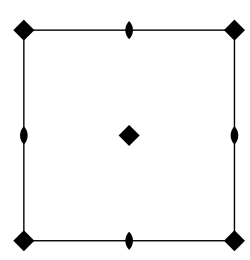

Typ $P 4$

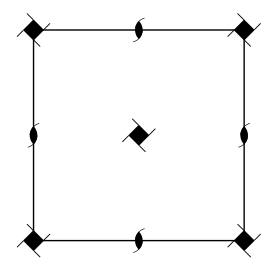

Typ $P 4_{1}$

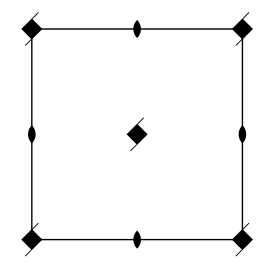

Typ $\mathrm{P}_{2}$

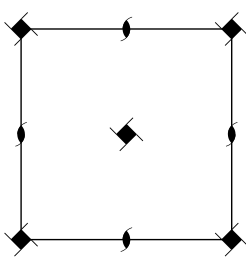

Typ $\mathrm{P}_{3}$

Fig. 5

Die Ecken der gezeichneten 4 Quadrate sind der Ursprung und $b_{1}, b_{1}+b_{2}$ sowie $b_{2}$. Das Diagramm ganz links illustriert die Achsenverteilung für $k=0$ : es zeigt, dass die Lote in den 4 Ecken und im Mittelpunkt des Quadrates Drehachsen sind. Das nächste Diagramm gehört zu $k=1$ : an Stelle der Drehachsen finden sich nun Achsen von Schraubungen der Ganghöhe $\frac{1}{4} b_{3}$. Die letzten zwei Diagramme veranschaulichen die Fälle $k=2$ und $k=3$ : die Schraubungen haben die Ganghöhen $\frac{2}{4} b_{3}$ und $\frac{3}{4} b_{3}$, was durch die andere Art der Flügelchen angedeutet ist.

In den Diagrammen sind auch die Positionen von Achsen, die von der Achse $U$ der Drehung $\varphi^{2}$ herrühren, eingezeichnet. Die Durchstosspunkte dieser Schar von Achsen mit der Ebene $W$ bilden das Gitter $\left(\|-\varphi^{2}\right)_{\mid W}^{-1}(\Gamma \cap W)$; es ist quadratisch und gleich $\left(\frac{1}{2} \Gamma\right) \cap W$. Für $k \in\{0,2\}$ sind die Geraden Drehachsen, für $k \in\{1,3\}$ Achsen von Schraubungen.

Die Dispositionen der Achsen sind in den 4 Fällen deutlich unterschiedlich. Dies zeigt, dass es 4 Typen von Raumgruppen in der arithmetischen Klasse P4 gibt. Die Kristallographen bezeichnen sie mit $P 4, P 4_{1}, P 4_{2}$ und $P 4_{3}$.

Im zweiten Fall ist $\Gamma$ gleich $\Gamma_{1} \cup \Gamma_{1}+\frac{1}{2}\left(b_{1}+b_{2}+b_{3}\right)=\Gamma_{1}+\mathbb{Z} \frac{1}{2}\left(b_{1}+b_{2}+b_{3}\right)$. Setze $\bar{\Gamma}=\pi_{W}(\Gamma)=\mathbb{Z} b_{1}+\mathbb{Z} b_{2}+\mathbb{Z} \frac{1}{2}\left(b_{1}+b_{2}\right)$; dann ist $L_{\varphi}^{-1}(\bar{\Gamma})$ das quadratische Gitter $\mathbb{Z} \frac{1}{2} b_{1}+\mathbb{Z} \frac{1}{2} b_{2}$. Die Anordnungen der Achsen für $v_{\varphi}=\frac{k}{4} b_{3}$ und $k \in\{0,1,2,3\}$ sind in Figur 6 dargestellt. Das Diagramm ganz links zeigt die Anordnung für $k=0$ : die Lote in den Ecken und in der Mitte des Quadrates sind Drehachsen, die anderen Lote sind Schraubenachsen mit der Ganghöhe $\frac{1}{2} b_{3}$ Das zweite Diagramm zeigt die Situation für $k=1$ : alle Lote in den Punkten von $L_{\varphi}^{-1}(\bar{\Gamma})$ sind Achsen von Schraubungen, deren 
Ganghöhen teils $\frac{1}{4} b_{3}$, teils $\frac{3}{4} b_{3}$ betragen. Geht man von $v_{\varphi}=\frac{1}{4} b_{3}$ zu $v_{\varphi}=\frac{2}{4} b_{3}$ über, werden die Lote in den Ecken und in der Mitte Schraubenachsen mit der Ganghöhe $\frac{1}{2} b_{3}$, die anderen Lote aber sind Drehachsen. Vergleicht man nun das erste und das dritte Diagramm, erkennt man, dass ihre periodischen Fortsetzungen durch eine Translation auseinander hervorgehen. Dies bedeutet, dass die dritte Gruppe durch Verschieben des Ursprunges in die erste Gruppe überführt werden kann. Ebenso sind die Gruppen des zweiten und vierten Diagrammes affin äquivalent. Daher gibt es in der arithmetischen Klasse I4 nur zwei eigentlich affine Typen von Raumgruppen; in der Kristallographie werden sie mit $I 4$ und $I 4_{1}$ bezeichnet.

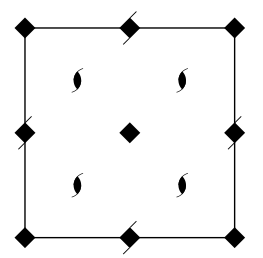

Typ I4

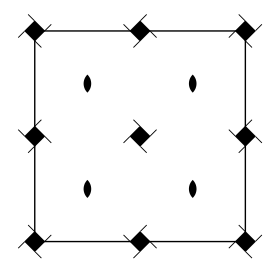

Typ $I 4_{1}$

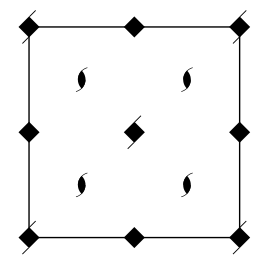

Typ I4

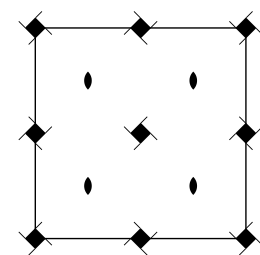

Typ $I 4_{1}$

Fig. 6

Bemerkungen. 1) Die oben vorgebrachten Überlegungen finden sich sinngemäss alle im Schoenfliesschen Werk [13]. Naturgemäss überzeugen sie umso eher, als man gewohnt ist, geometrisch, und nicht rechnerisch, zu argumentieren.

2) Die Analyse der Anordnung der Symmetrieelemente einer Raumgruppe $G$ kann auf die Analyse kleinerer Gruppen abgestützt werden; denn es gilt: Sei G eine Raumgruppe mit Gitter $\Gamma$ und Punktgruppe $G_{0}$. Für jede Untergruppe $H \subset G_{0}$ ist das Urbild von $H$ unter der Projektion $G \rightarrow G_{0}$ eine Untergruppe $G_{H}$ von $G$ mit Gitter $\Gamma$ ([13], Cap. VI, Lehrsatz XVI). Schoenflies benützt dieses Resultat bei den Untersuchungen der Raumgruppen mit nicht zyklischer Punktgruppe ausgiebig. Will man mit seiner Methode die Raumgruppen einer solchen arithmetischen Klasse finden, hat man daher auch die Raumgruppen von anderen Klassen zu ermitteln. Beim Burckhardtschen Zugang ist dies im Prinzip nicht nötig (s. [14], Nummer 6.5).

\subsection{Konstruktion der Raumgruppen nach Burckhardt}

Seien $\Gamma$ das Gitter $\mathbb{Z}^{3} \subset \mathbb{R}^{3}$ und $H$ die Gruppe der Ordnung 4, die von den Potenzen der Drehung $\varphi$ : $\left(x_{1}, x_{2}, x_{3}\right)^{t} \mapsto\left(-x_{2}, x_{1}, x_{3}\right)^{t}$ gebildet wird. Jede Raumgruppe $G$ von $\left(\mathbb{R}^{3},\langle-,-\rangle\right)$, welche der arithmetischen Klasse von $\left(\mathbb{Z}^{3}, H\right)$ angehört, wird vom Gitter der Translationsvektoren $\mathbb{Z}^{3}$ und einem Element der Form $(v, \varphi)$ mit $v \in \mathbb{R}^{3}$ erzeugt. Es stellen sich zwei Fragen:

(i) Welche Vektoren $v$ sind zugelassen?

(ii) Wann liefern Vektoren $v, v^{\prime}$ eigentlich affin äquivalente Gruppen?

Die Antwort auf (i) ergibt sich wie bei Schoenflies: $(v, \varphi)^{4}$ ist eine Translation; sie gehört genau dann zu $G$, falls $v+\varphi(v)+\varphi^{2}(v)+\varphi^{3}(v)=\left(0,0,4 v_{3}\right)^{t}$ in $\mathbb{Z}^{3}$ liegt, d.h., falls $v_{3} \in \frac{1}{4} \mathbb{Z}$ ist. Um den ersten Teil von (ii) zu beantworten, imitiert man das 
geometrische Verfahren des Verschiebens des Ursprunges mit algebraischen Mitteln: dies führt auf die Translationsklassen (s. [14], Nummer 6.2). Durch Übergang von $G$ zu einer andern Gruppe in der gleichen Translationsklasse kann man erreichen, dass der Vektor in $(v, \varphi)$ die Form $\left(0,0, \frac{k}{4}\right)^{t}$ mit $k \in \mathbb{Z}$ bekommt. Es folgt, dass jede Raumgruppe der arithmetischen Klasse $P 4$ eigentlich affin äquivalent ist zu einer Gruppe, die von $\mathbb{Z}^{3}$ und einem Element der Form $\left(\frac{k}{4}, \varphi\right)$ mit $k \in\{0,1,2,3\}$ erzeugt wird. Es bleibt die Frage, ob verschiedene Werte von $k$ auf eigentlich affin äquivalente Gruppen führen können. Man kann sie beantworten, indem man die Wirkung des Normalisators $N_{\mathrm{SL}(3, \mathbb{Z})}(H)$ auf der Menge der Translationsklassen analysiert (s. [14], Nummer 6.4).

\section{Literatur}

[1] Bieberbach, L.: Über die Bewegungsgruppen der Euklidischen Räume, Teil I. Math. Annalen 70 (1911), 297-336.

[2] Bieberbach, L.: Über die Bewegungsgruppen der Euklidischen Räume, Teil II. Math. Annalen 72 (1912), 400-412.

[3] Brown, H.; Bülow, R.; Neubüser, J.; Wondratschek, H.; Zassenhaus, H.: Crystallographic groups of four-dimensional space. John Wiley \& Sons, 1978.

[4] Burckhardt, J.J.: Bemerkungen zur arithmetischen Berechnung der Bewegungsgruppen. Comment. Math. Helv. 2 (1930), 91-98.

[5] Burckhardt, J.J.: Zur Theorie der Bewegungsgruppen. Comment. Math. Helv. 6 (1934), 159-184.

[6] Burckhardt, J.J.: Bewegungsgruppen in mehrdimensionalen Räumen. Comment. Math. Helv. 9 (1936/37), 284-302.

[7] Burckhardt, J.J.: Die Bewegungsgruppen der Kristallographie. Birkhäuser, 1947 (1. Auflage) und 1966 (2. neubearbeitete Auflage).

[8] Buser, P.: A geometric proof of Bieberbach's theorems on crystallographic groups. L'Enseignement Mathématique 31 (1985), 137-145.

[9] Fedorov, E.S.: Zusammenstellung der krystallographischen Resultate des Herrn Schoenflies und der meinigen. Zs. f. Kristallogr. 20 (1892), 25-75.

[10] Hilbert, D.: Mathematische Probleme. Ges. Abh. Bd. 3, 290-329.

[11] International Tables for Crystallography. Volume A on space-group symmetry (T. Hahn); Kluwer Academic Publishers 2002 (fifth edition).

[12] Plesken, W.; Schulz, T.: Counting crystallographic groups in low dimensions. Exp. Math. 9 (2000), $407-411$.

[13] Schoenflies, A.: Krystallsysteme und Krystallstructur. Teubner, 1891.

[14] Strebel, R.: Burckhardtsche Bestimmung der Raumgruppen II. Erscheint in Elem. Math..

\section{Ralph Strebel}

Departement für Mathematik

Universität Freiburg

CH-1700 Freiburg, Schweiz

e-mail: ralph.strebel@unifr.ch 